present. Accordingly, coaches play a crucial role in recognising the signs and symptoms of concussion, and ensuring that players with a suspected concussion are managed correctly. Coaches have also been found to play a central role in reinforcing or undermining a sport culture in which athletes report injuries, including concussion. The aim of this study was to assess perceived ability to assess and manage concussion; and to explore, coaches' communication practices about concussion safety with their players.

Methods Using a self-report questionnaire data were captured on coaches' communication practices about concussion safety and perceived ability to assess and manage concussion among their athletes. Data was collected electronically from June-September 2015. Results were analysed using SPSS Version 22.

Results The final sample consisted of 106 coaches (59 male, 37 female) from the island of Ireland. Results showed that a majority of coaches did not feel equipped to promote concussion education among their athletes (76.5\%), determine if a player has sustained a concussion (69.7\%) or to help an athlete who obtained a suspected concussion during training or a match (62.6\%). Additionally, before the season, a majority of coaches did not talk to their athletes about concussion safety and the importance of concussion reporting.

Conclusions Findings from this study suggest that interventions should encourage communication about concussion safety with athletes and should provide coaches with communication skills and strategies about how to do this. Additionally, coach targeted interventions should focus on instructing coaches on how to recognise and manage a suspected concussion in one of their athletes.

\section{UNDERSTANDING MENTAL HEALTH SYMPTOMS SUFFERED BY TRAUMATIC BRAIN INJURY PATIENTS IN MOSHI, TANZANIA}

\footnotetext{
1,2 João Ricardo Nickenig Vissoci, ${ }^{3}$ Joan Kwesigabo, ${ }^{3}$ Green Geffrey, ${ }^{3}$ Mark Mvungi, ${ }^{2}$ Catherine Staton. ${ }^{1}$ Faculdade Ingá, Brazil; ${ }^{2}$ Duke University, US; ${ }^{3}$ Kilimanjaro Christian Medical Centre, Tanzania
}

\subsection{6/injuryprev-2016-042156.685}

Background Traumatic Brain Injury (TBI) is the most common cause of injury death and disability globally. While TBI increases mental health burden, there is no current literature in low and middle-income countries (LMIC) about these patients. This project evaluated the pre-injury mental health of TBI at the Kilimanjaro Christian Medical Centre (KCMC) in Moshi, Tanzania.

Methods Surveys were conducted of TBI patients between May September 2015. Participants were $>18$ years old and responded at discharge the questionnaires: PHQ-9, Kessler, CES-D, and AUDIT. Data were descriptively represented and questionnaires classified according to the cut-offs: PHQ-9 >4, CES >15, Kessler $>20$, and AUDIT $>7$. Frequences, means with standard deviations (sd) were reported and a spearman correlation was used to evaluate associations.

Results Of all 77 TBI patients, most are male (84\%) with mean age of 35 (sd 13) and married (61\%). Patients were mainly farmers, skilled or unskilled workers or work in business. While a small percentage of patients have signs of depression $(2.6 \%$ to $9.1 \%)$ and anxiety (4.2\%) prior to their injury, a significant proportion have harmful or hazardous drinking behaviour (42.9\%). A moderate correlation was observed between depression and anxiety symptoms $(\mathrm{R}=0.44$ and $\mathrm{R}=0.51)$. A small and not significant association was found between hazardous or harmful drinking and depression $(\mathrm{R}=-0.02$ and $\mathrm{R}=-0.09)$ or anxiety $(\mathrm{R}=0.02)$ amongst this population.

Conclusions This is the first report of pre-injury mental health of TBI patients in Tanzania. Although significant proportions of patients did not show large mental health systems hazard or harmful alcohol use was concerningly high. However, association patterns demonstrated that patients with harmful or hazardous drinking are not the same with depression and anxiety score. This is an important preliminary finding to understand the baseline mental health status of our TBI patients in a low income setting.

\section{STUDY OF PREDICTORS OF PROGNOSIS OF MILD HEAD INJURY}

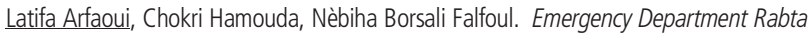

10.1136/injuryprev-2016-042156.686

Background The mild head injury is a mild traumatic pathology in most cases and the role of emergencies often comes down to an early lesion assessment, analgesic treatment and practice of a brain scan. The follow up is assured by the neurology departments and no feedback is communicated to emergencies and thus no relationship between the original picture and the prognosis can be established. The aim of our study is to evaluate the prognosis of mild head injury after one month based on initial data. Methods Descriptive prospective study of the clinical and evolutionary characteristics of mTBI isolated over a three months period (October-December 2009).

Results We collected 200 cases of mTBI. The average age of patients was 38.9 years with a sex ratio of 1.78 . The initial loss of consciousness was reported in 95 patients (47.5\%) and was predictive of the presence of intracranial lesions $(p=0.02)$. Follow up has been done in $84.5 \%$ of cases. CT scan was performed in $50.5 \%$ and allowed to reveal traumatic defects in $16.8 \%$ of cases. The existence of an epistaxis, agitation or periorbital hematoma was correlated with the existence of traumatic injuries to the scanner $(\mathrm{p}<0.05) .169$ patients were evaluated at one month by the Glasgow Outcome Scale (GOS). 50\% of patients kept variable somatic complaints (headache, irritability, insomnia ...). GCS, agitation, CCEP, CT score, initial loss of consciousness, Masters score and the ISS score are prognostic indicators.

Conclusions The initial loss of consciousness, the initial GCS, evaluating the severity of the accident and the patient's condition are predictors of the existence of intracranial lesions indicating brain scan. These factors with brain damage, Masters Score and ISS are prognostic factors which are correlated with the appearance of post concussion syndrome. Early psychological treatment is necessary.

\section{Child and Adolescent Safety}

\section{Post Tue 2.8}

\section{ASSAULT RELATED MUSCULOSKELETAL INJURIES IN ADOLESCENTS AT ER}

Kyaw Zeyar Tun, Aung Thein Htay, Thit Lwin. Orthopaedic and Trauma Department, University of Medicine 1, Yangon

10.1136/injuryprev-2016-042156.687 
Background Adolescent is a time of heightened violence and the frequency of engaging in violent behaviour is greater for adolescents than for all other age groups. A better understanding of factors related to assault among adolescents is important to reduce the incident. This study described and characterised the sociodemographic factors, circumstances and characteristic of assault in emergency department.

Methods A descriptive study was carried on 140 patients at ER, Yangon General Hospital during one year. Adolescent assault victims with musculoskeletal injuries were collected involving demographic details, circumstances of injury, characteristics of injury and initial treatment parameter.

Results Among the 140 respondents, males (80\%) and females (20\%) reported been an assault. Males were more likely to have been in a physical fight. The majority of cases $(47.1 \%)$ were of low educational status (primary education). Casual workers and dependent were the most common group among the injured, occurred at public place during night time. Blunt object (81\%) were the most common weapons used. Head and neck were commonly affected in over half of cases (58\%) and soft tissue injuries (90\%) were the most frequent type. $71 \%$ of the patients were treated as out-patients.

Conclusion assaults are responsible for significant morbidity and may be an important opportunity for prevention. Socioeconomic status, contributing factors and injury characteristics identified to this study should help strategies for preventing adolescent assault.

\section{CHARACTERISTICS OF UNINTENTIONAL AND INTENTIONAL CHILD INJURIES FROM EMERGENCY AND OUTPATIENT ROOM IN CHINA, 2006-2014}

Wang Yuan, Ye Pengpeng, Duan Leilei. National Centre for Chronic and Noncommunicable Disease Control and Prevention, Chinese Centre for Disease Control and Prevention

\subsection{6/injuryprev-2016-042156.688}

Background Injury is the first death cause of children aged 0 to 17 in China. Prevention of unintentional and intentional injury was totally different.

Methods Data collected from National Injury Surveillance System that based on emergency and outpatient department of 126 sentinel hospitals around the whole country through 2006 to 2014 were analysed. Mechanism, place where injury occurred, severity and outcome of unintentional and intentional injuries among patients aged 0 to 17 were summarised.

Results Among the 895,243 patients aged 0-17, 94.22\% cases were unintentional injuries; $4.84 \%$ cases were intentional injuries (interpersonal violence cases: $4.50 \%$, self-injuries: $0.34 \%$ ).

The top injury mechanisms were falling (51.95\%), road traffic injury (14.36\%), blunt injury (9.59\%), animal injury $(9.11 \%)$ and sharp injury (6.88\%) among unintentional injuries; blunt injury $(63.73 \%)$, sharp injury (12.01\%), animal injury (7.94\%), falling $(4.35 \%)$, poisoning $(0.56 \%)$ among interpersonal violence cases; poisoning (51.96\%), sharp injury (21.29\%), falling $(10.60 \%)$, blunt injury $(5.66 \%)$, road traffic injury $(3.70 \%)$ among self-injuries.

$40.35 \%$ of unintentional injuries occurred in home, followed by road (19.53\%), school and public place (17.63\%), public housing (15.50\%); for interpersonal violence cases, $47.64 \%$ occurred in school and public place, followed by public housing (19.60\%), home (10.69) and road (8.95\%); for self-injuries, $65.08 \%$ occurred in home, followed by school and public place (12.79\%), public housing (10.04\%) and road (3.63\%).

Among unintentional injuries, $81.62 \%$ were mitigated injuries, $17.21 \%$ were moderate injuries and $1.16 \%$ was severe injuries. The proportion of mitigated, moderate and severe injuries among interpersonal violence cases and self-injuries were $79.53 \%$, $18.88 \%, 1.59 \%$ and $51.55 \%, 38.36 \%, 10.08 \%$ respectively.

$86.42 \%$ of unintentional injuries were discharged, $12.96 \%$ were hospitalised and $0.09 \%$ died; the proportion of being discharged, being hospitalised and dying among interpersonal violence cases and self-injuries were $80.16 \%, 18.96 \%, 0.08 \%$ and 54.32\%, 43.98\% , 1.08\% respectively.

Conclusions It's necessary to have a good understanding of the characteristics of child injuries for child injury prevention.

\section{BRITISH PARENTS' SELF-REPORTED ROAD SAFETY BELIEFS, PRACTICES AND RULES}

'Karen Pfeffer, ${ }^{2}$ Zahra Tabibi. ${ }^{1}$ University of Lincoln, UK; ${ }^{2}$ Ferdowsi University of Mashhad, Iran

\subsection{6/injuryprev-2016-042156.689}

Background Children are at high risk for pedestrian deaths and injury (World Health Organisation, 2009). Parents play an important role in teaching children pedestrian skills in context. The aims of this research were to investigate parents' self-reported beliefs, practices and road safety rules when crossing streets with their children.

Methods A questionnaire was administered to parents of 234 children aged $3-10$ years $($ mean $=6.23$ years, $S D=1.83)$. Participants were parents of children who were attending a university children's event in a small British city. The majority of respondents were mothers. Questions were about pedestrian experiences, safety rules and risks, also who they think is best suited to teach children about road safety.

Results The majority (61.5\%) normally walked to school on a daily basis. The age parents thought it was appropriate for boys and girls to walk to school alone was significantly lower than the appropriate age to walk to school with peers. Parents ranked themselves as the most suited to teach children about road safety in comparison to professionals such as teachers and road safety education officers. Only 3 parents said that they did not have any specific road safety rules and the majority of parents reported that they hold their child's hand when crossing the road. The road safety rules that parents reported were similar across child age groups.

Conclusions Our sample of parents reported that they walk with their children frequently. Parents' self-reported rules and behaviour did not differ with child age. The younger age at which British parents considered it appropriate for children to walk to school with peers compared to alone needs further investigation. In this respect, further research should consider comparisons of road risk and 'stranger danger' (abduction risk). Also, further research should focus on reasons that parents considered road safety professionals less suited to teach children about road safety than parents. 